\title{
Comparação entre os resultados da descompressão orbitária antro-etmoidal isolada e associada à remoção de tecido adiposo
}

\author{
A comparative study between the results of antro-ethmoidal orbital decompression \\ isolated and associated with orbital fat removal
}

\author{
Allan Christian Pieroni Gonçalves \\ Frederico Castelo Moura ${ }^{2}$ \\ Janete P. Moura ${ }^{3}$ \\ Walter Bloise $^{4}$ \\ Mario Luiz Ribeiro Monteiro ${ }^{5}$
}

Trabalho realizado na Divisão de Clínica Oftalmológica do Hospital das Clínicas da Faculdade de Medicina da Universidade de São Paulo.

Pós graduando da Faculdade de Medicina da Universidade de São Paulo (USP). São Paulo (SP).

${ }_{2}^{2}$ Pós graduando da Faculdade de Medicina da USP. São Paulo (SP).

Médica assistente do Departamento de Endocrinologia do Hospital das Clínicas da Faculdade de Medicina da USP. São Paulo (SP).

${ }^{4}$ Professor Livre Docente do Departamento de Endocrinologia do Hospital das Clínicas da Faculdade de Medicina da USP. São Paulo (SP)

${ }^{5}$ Professor Livre Docente da Clínica Oftalmológica do Hospital das Clínicas da Faculdade de Medicina da USP. São Paulo (SP).

Endereço para correspondência: Allan C. Pieroni Gonçalves - Rua Indiana 337 apto 82 - São Paulo (SP) CEP 04562-000

E-mail: mlrmonteiro@terra.com.br

Recebido para publicação em 18.11.2004 Aprovação em 04.03.2005

Nota Editorial: Após concluída a análise do artigo sob sigilo editorial e com a anuência do Dr. Roberto Abucham sobre a divulgação de seu nome como revisor, agradecemos sua participação neste processo.

\section{RESUMO}

Objetivo: Comparar os resultados da descompressão orbitária antroetmoidal isolada àqueles da mesma cirurgia associada à remoção de tecido orbitário em paciente com orbitopatia distiroidiana seqüelar. Métodos: Foram analisadas 2 modalidades de descompressão orbitária. Ambas tiveram como indicação apenas a correção estética e do desconforto ocular dos pacientes. Todos pacientes se apresentavam na fase inativa da orbitopatia distiroidiana. Em um grupo de 12 pacientes (grupo 2) foram realizadas 19 cirurgias de descompressão orbitária antro-etmoidal isolada. No outro grupo (grupo 1) composto por 8 pacientes foram realizadas 10 cirurgias de descompressão óssea antro-etmoidal associada à remoção de aproximadamente 1 centímetro cúbico de tecido adiposo da órbita. Os resultados das cirurgias nos dois grupos foram comparados entre si. Resultados: No grupo 2 a redução da proptose oscilou entre 1 e $5 \mathrm{~mm}$ (média $3,68 \pm 1,10 \mathrm{~mm}$ ) e no grupo 1 variou de 1 a $5 \mathrm{~mm}$ (média 3,25 $\pm 1,36 \mathrm{~mm}$ ). Conclusões: Tanto a descompressão óssea isolada como aquela associada à remoção de tecido adiposo orbitário apresentam redução semelhante na proptose, não havendo diferença significativa entre elas. A remoção de gordura temporal inferior, em torno de 1 centímetro cúbico parece não aumentar a redução da proptose quando comparada com a cirurgia descompressiva óssea isolada. Porém fatores como a complacência de tecidos moles, a quantidade de gordura retirada e as janelas ósseas realizadas em cada grupo devem ser considerados na conclusão deste trabalho retrospectivo de comparação entre técnicas.

Descritores: Doença de Graves; Descompressão cirúrgica/métodos; Órbita/cururgia; Osso etmóide/cirurgia; Lipectomia; Estudo comparativo; Estudos retrospectivos

\section{INTRODUÇÃO}

A orbitopatia distireoidiana é uma doença inflamatória que afeta a órbita e tecidos perioculares. Corresponde a uma das mais freqüentes afecções da órbita acometendo principalmente pacientes entre 20 e 60 anos. Inúmeras alterações funcionais e estéticas caracterizam esta doença como o exoftalmo, retração palpebral, estrabismo, congestão palpebral e conjuntival, exposição corneana e neuropatia óptica compressiva. O seu tratamento é complexo e envolve além da abordagem clínica, procedimentos cirúrgicos e radioterápicos. A descompressão orbitária é um destes procedimentos cirúrgicos que pode ser indicado tanto na fase aguda da doença, quando 
ocorre neuropatia óptica ou exposição corneana grave, como na fase crônica para corrigir deformidades seqüelares cosméticas ou funcionais ${ }^{(1)}$.

A descompressão orbitária pode ser realizada através da remoção de paredes orbitais (descompressão óssea) ou através da remoção de tecido adiposo da órbita ${ }^{(2-3)}$. Inúmeras são as técnicas descritas para a descompressão óssea, podendo envolver a remoção de uma a quatro paredes orbitais, dependendo da gravidade da exoftalmia. A maioria dos autores utiliza a remoção parcial de 2 ou 3 paredes orbitais incluindo as paredes inferior, medial e lateral da órbita ${ }^{(4)}$. Uma outra técnica de descompressão orbitária através da remoção de grande quantidade de tecido adiposo da órbita, com o objetivo de diminuição do conteúdo orbitário ao invés do aumento do continente, foi descrita pela primeira vez em $1988^{(5)}$. Embora este procedimento como descrito originalmente não seja utilizado pela maioria dos cirurgiões de órbita, o trabalho foi importante para introduzir a idéia da remoção de tecido adiposo como elemento coadjuvante na descompressão orbitária.

Em um trabalho prévio relatamos nossa experiência em 22 órbitas operadas utilizando uma técnica de descompressão orbitária óssea relativamente conservadora, com remoção de parte das paredes inferior e medial da órbita ${ }^{(6)}$. Nos últimos anos passamos a associar a tal procedimento a remoção de tecido adiposo da região temporal inferior da órbita. O objetivo do trabalho atual é comparar os resultados da cirurgia de descompressão orbitária antro-etmoidal isolada com aqueles obtidos com a mesma cirurgia associada à remoção de tecido adiposo da órbita.

\section{MÉTODOS}

No período de janeiro de 1992 a março de 1998, 14 pacientes portadores de doença de Graves foram submetidos à descompressão orbitária antro-etmoidal óssea isolada. Dois pacientes ( 3 cirurgias) foram operados na fase aguda (congestiva) da doença e os demais (12 pacientes, 19 órbitas) foram operados na fase não inflamatória ${ }^{(6)}$. Os 19 pacientes (grupo 2) operados na fase não inflamatória da doença foram comparados com 8 pacientes (10 órbitas) operadas de novembro de 1998 a dezembro de 2002 no Hospital das Clínicas da Faculdade de Medicina da Universidade de São Paulo (grupo 1). Nestes últimos a descompressão óssea foi associada à remoção de tecido adiposo da órbita.

Desta última série a orbitopatia foi bilateral em 5 casos e unilateral em 3. A cirurgia foi unilateral em 6 pacientes. Em 4, embora a proptose fosse bilateral, julgou-se necessária a descompressão apenas de uma órbita. Dois pacientes já haviam sido operados em um dos olhos. Em apenas um paciente a cirurgia foi bilateral e simultânea (paciente 3 ).

A cirurgia foi realizada por via transconjuntival segundo técnica previamente descrita $^{(7)}$. Sob anestesia geral e após infiltração de xilocaína com adrenalina realiza-se uma cantotomia lateral e lise do ligamento cantal lateral inferior. A pálpebra inferior liberada é tracionada com fio de seda 4-0 e a conjuntiva incisada 6 a $8 \mathrm{~mm}$ abaixo do tarso, do canto lateral para o medial até a região da carúncula. Utiliza-se retrator maleável para deslocar o globo ocular para cima e afastadores são colocados na incisão conjuntival inferior, de tal forma a esticar os retratores da pálpebra sobre o rebordo orbitário. A fáscia capsulopalpebral é aberta expondo-se o periósteo. Move-se o retrator maleável de tal forma a afastar também o músculo oblíquo inferior e protegê-lo juntamente com o globo ocular. O periósteo é incisado ao longo de toda a extensão do rebordo orbitário. Afasta-se o periósteo descolado com retrator e disseca-se todo o assoalho orbitário com descolador de periósteo e identifica-se o nervo infra-orbitário. Realiza-se uma abertura na parede inferior da órbita de preferência no seu setor mais medial, com osteótomo, criando-se uma comunicação com o seio maxilar. Remove-se toda a parede inferior na porção medial ao nervo infra-orbitário, até a parede posterior do seio maxilar. A parede inferior lateral ao nervo infra-orbitário não foi removida em nossos pacientes. Removemos também a parede medial abaixo do forame das artérias etmoidais anterior e posterior. Completada a remoção óssea e controlado o sangramento da mucosa sinusal, procede-se à incisão da periórbita (duas ou mais) no plano sagital, idealmente colocadas entre os músculos retos. Incisões transversais também são realizadas de tal forma que se observe herniação da gordura para os seios maxilar e etmoidal.

Após a descompressão óssea antro-etmoidal, foi retirado da região temporal inferior da órbita tecido adiposo com volume total em torno de $1 \mathrm{ml}$ de gordura orbitária medido em tubo cilíndrico de $10 \mathrm{ml}$ com graduação volumétrica. A conjuntiva é suturada com fio absorvível 5-0 ou 6-0 e o tendão cantal lateral é suturado ao periósteo com fio inabsorvível 5-0. A borda palpebral é recomposta com pontos simples de fio inabsorvível 6-0 na linha tarsal, linha cinzenta e linha dos cílios. A pele é suturada com fio de nylon 6-0 $0^{(3,6-7)}$.

\section{RESULTADOS}

A tabela 1 mostra a idade, sexo, exoftalmometria pré-operatória, exoftalmometria pós-operatória e redução do exoftalmo resultante dos pacientes submetidos à descompressão orbitária transetmoidal associada à retirada de tecido adiposo (grupo 1). Apenas um paciente era do sexo masculino e os demais 7 do sexo feminino. A idade dos pacientes variou entre 27 e 60 anos (média de 41,7 anos). Todos os pacientes tiveram a indicação primariamente estética embora em vários deles a proptose importante também ocasionasse desconforto ocular com tipo ardor e sensação de corpo estranho devido à exposição corneana, a despeito de tratamento lubrificante. Nas 10 órbitas analisadas correção da proptose variou entre 1 e $5 \mathrm{~mm}$, com média e desvio padrão de 3,25 $\pm 1,36 \mathrm{~mm}$.

Quanto às complicações, pudemos observar apenas perda transitória da sensibilidade na região do nervo infra-orbitário. No entanto, nenhum paciente apresentou perda da sensibilidade 


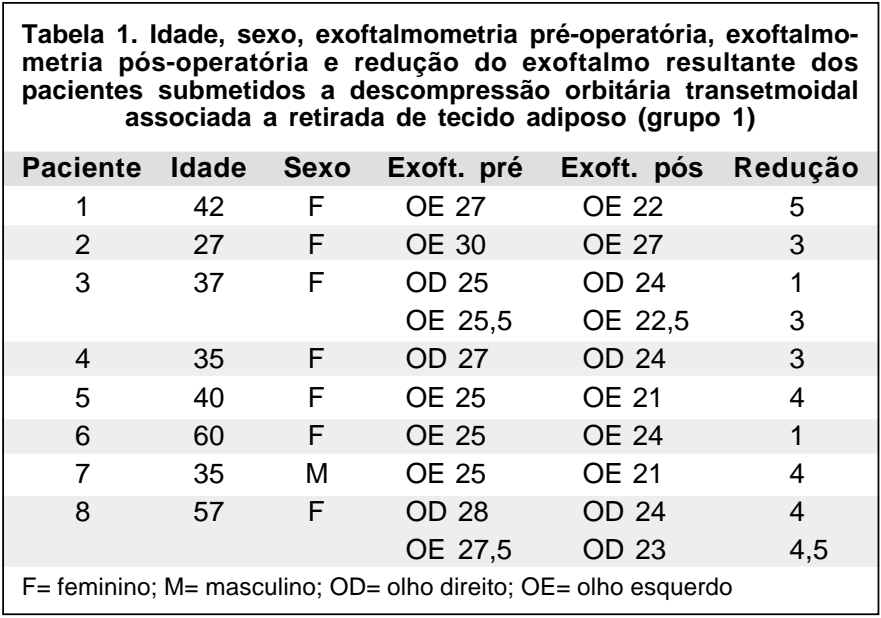

definitiva, tendo o sintoma desaparecido após algumas semanas. Nenhum paciente desenvolveu diplopia na posição primária do olhar ou na posição de leitura que necessitasse de cirurgia. $\mathrm{O}$ caso 6, que apresentou pequena redução da proptose, necessitou de nova cirurgia de descompressão, realizada via transcaruncular para descompressão de parede medial posterior.

A tabela 2 mostra idade, sexo, exoftalmometria pré e pósoperatória dos 12 pacientes (19 cirurgias) operados através da descompressão óssea isolada, sem remoção de tecido gorduroso orbitário evidenciando melhora importante da proptose (grupo 2). A redução (média \pm desvio padrão) da proptose nesses pacientes foi de $3,68 \pm 1,10 \mathrm{~mm}$, oscilando entre 1 e $5 \mathrm{~mm}$. Dos 12

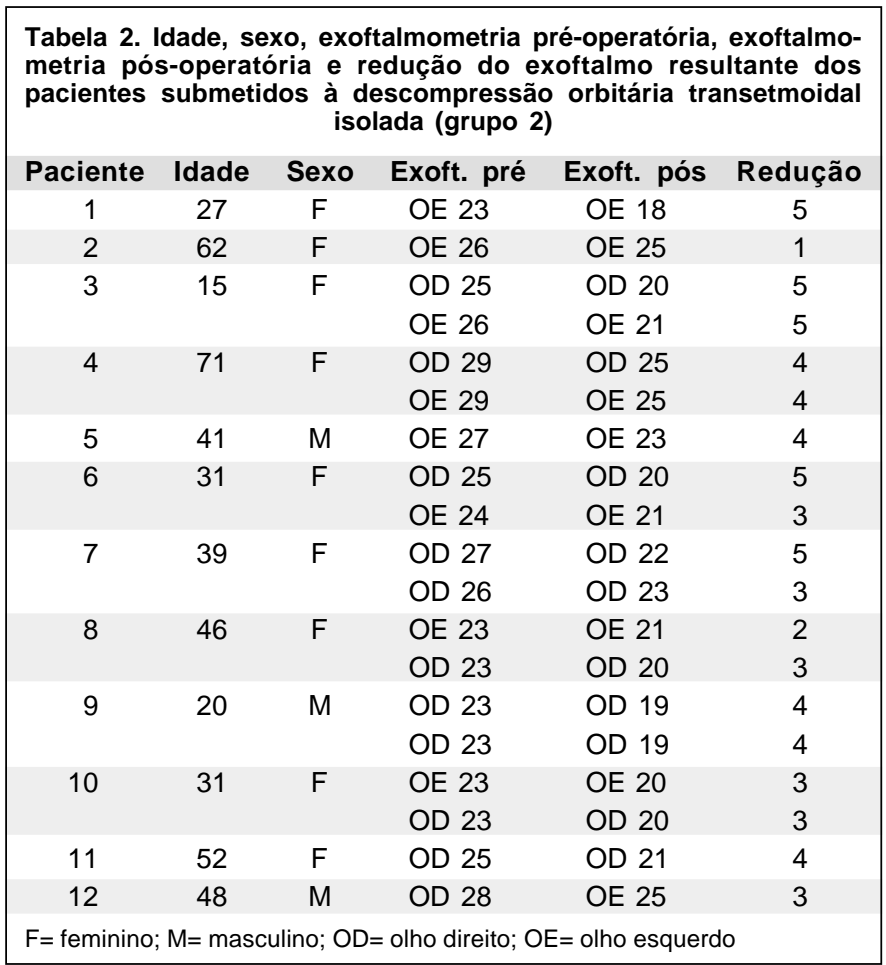

pacientes 3 pacientes eram do sexo masculino. A idade dos pacientes variava de 15 a 71 anos de idade (média de 40,82 anos). As complicações e dificuldades foram discutidas no trabalho anterior ${ }^{(6)}$.

O estudo estatístico, utilizando-se o teste t de Student, não revelou diferença significativa entre os resultados da cirurgia descompressiva isolada e aquela associada à remoção de tecido adiposo orbitário $(\mathrm{p}=0,36)$.

\section{DISCUSSÃO}

Desde a primeira descrição de descompressão orbitária por Dollinger em $1911^{*}$, uma série de técnicas cirúrgicas foi desenvolvida para aumentar a capacidade da cavidade orbitária ou diminuir o volume das partes moles no tratamento da orbitopatia distireoidiana ${ }^{(8)}$. Subseqüentes modificações visaram melhorar resultados e simplificar a cirurgia. Sendo que estudos comparativos são instrumentos científicos na busca deste aperfeiçoamento.

A orbitopatia distireoidiana é uma patologia com quadro clínico radiológico variável podendo, segundo alguns autores, ser dividida independente da atividade em três subgrupos: o primeiro com aumento volumétrico de músculos extraoculares e gordura; o segundo com aumento volumétrico apenas dos músculos extra-oculares; e o terceiro, forma lipogênica, com aumento volumétrico do tecido adiposo somente ${ }^{(9-10)}$. A escolha da técnica cirúrgica empregada, portanto, deve ser determinada pela característica da orbitopatia, pela quantidade de descompressão proposta assim como pela preferência do cirurgião.

Dentre as várias abordagens cirúrgicas existentes para descompressão orbitária, as que não envolvem cicatriz aparente, como a transcaruncular, transconjuntival, lateral subcantal, e coronal têm atraído maior interesse devido à importância de se associar bons resultados funcionais a estéti$\cos ^{(4,11-12)}$. Indicada em nosso serviço principalmente no tratamento de deformidades estéticas, a abordagem transconjuntival com descompressão antro-etmoidal segundo nossa experiência mostra-se bastante eficiente para reduzir a proptose e é associada a um baixo índice de complicações embora alguns autores reportem diferentes índices ${ }^{(13)}$. Em uma revisão dos resultados desta técnica de descompressão orbitária em um grande número de casos, observou-se baixa morbidade com um índice de apenas 5,7\% de diplopia induzida ${ }^{(14)}$.

A descompressão orbitária baseada apenas na remoção de tecido gorduroso reduz proptose com menor morbidade em relação à descompressão óssea. Uma revisão retrospectiva de 41 cirurgias desta modalidade de descompressão apresentou redução média de 4,7 mm com retirada com excisão de 7,31 1 ,9 $\mathrm{ml}$ de gordura extra e intraconal. Enquanto uma série adicional

\footnotetext{
* Dollinger (1911) apud ${ }^{(8)}$
} 
de 158 casos teve redução média de $1,8 \mathrm{~mm}$ da proptose ${ }^{(15-16)}$. Até no tratamento da neuropatia óptica da orbitopatia distireoidiana a descompressão de gordura têm sido utilizada com resultados satisfatórios ${ }^{(9)}$. Todas estas séries relatam baixos índices de complicações.

Em relação à descompressão de parede lateral da órbita a literatura apresenta uma significante variação de resultados cirúrgicos decorrentes principalmente pela grande variação de técnicas utilizadas. A remoção da rima e porção anterior da parede lateral da órbita oferece mínima expansão orbitária uma vez que o osso zigomático e a porção anterior da asa maior do osso esfenoidal não são espessos. Esta remoção apresenta também resultado estético questionável seja pela incisão na pele, seja por possível retração dos tecidos acima do arco zigomático removido, necessitando algumas vezes de reconstituição estética ${ }^{(6,17)}$. Entretanto, a parede lateral posterior desde sua porção inferior até o teto orbitário é composta por áreas ósseas espessas apresentando um significante potencial para expansão do volume orbitário ${ }^{(4,18)}$. Uma descompressão de parede posterior da órbita é muito mais eficiente em retro-deslocamento do bulbo ocular, porém a abordagem e técnica são de grande complexidade e morbidade. Para realizar esta descompressão são descritas tanto a abordagem transconjuntival de "swinging eyelid" ${ }^{(19)}$ como a abordagem coronal, como a maioria dos autores preconiza devido a necessidade de visualização de procedimento de tal complexida$\mathrm{de}^{(4,11,13,20)}$. A modelagem da parede posterior necessita do uso de brocas e a retirada de trechos ósseos expõe a dura mater da fossa cerebral anterior e medial. Alguns autores preconizam o uso deste tipo de descompressão como primeira escolha no tratamento da orbitopatia distireoidiana sem acometimento do nervo com incorporação de paredes adicionais se necessário $^{(21)}$. Possíveis complicações da descompressão de parede lateral posterior com abordagem coronal são: a fístula liquórica, ptose de supercílio pela paresia do músculo frontal, enfraquecimento de músculo temporal, alacrimia e oscilopsia vertical ao mastigar além de estrabismos ${ }^{(4)}$. $\mathrm{O}$ acesso coronal também tem sua desvantagem em relação ao aumento do tempo cirúrgico e de pós-operatório, além de complicações inerentes à técnica do cirurgião.

Existem muitas limitações relacionadas aos estudos retrospectivos comparando diferentes técnicas de descompressão orbitária. Vários fatores interferindo no resultado terapêutico como pré-tratamento, grau de envolvimento extra-ocular e duração da doença são geralmente não são geralmente randomizados. Cada técnica tem sua curva de aprendizado e diferentes cirurgiões também alteram os resultados obtidos.

Entendemos que para o tratamento das deformidades estéticas residuais da orbitopatia distireoidiana a cirurgia descompressiva deva ser analisada com parcimônia pesando muito bem as vantagens e riscos da descompressão. Uma vez que se podem associar outras medidas corretivas como a blefaroplastia e a correção da retração palpebral com bons resultados estéticos acreditamos que uma correção menor da proptose, com mais segurança, seja preferível quando comparado a ci- rurgias mais agressivas que estão associadas a maior risco de complicações. Ao se planejar uma maior redução da proptose dos pacientes, mantendo o conceito da menor agressividade, optamos por associar a retirada de gordura ao invés da fratura de parede lateral pois, conforme já discutido, a remoção da parede lateral anterior não apresenta bom resultado podendo ainda piorar a estética e a remoção da parede posterior prevê aumento em tempo de recuperação no pós-operatório além das possíveis complicações. A combinação de descompressão óssea com a retirada de gordura orbitária já foi descrita em trabalho prévio, porém sem a menção da quantidade de gordura retirada ${ }^{(21)}$.

\section{CONCLUSÃO}

No trabalho atual procuramos comparar o resultado da descompressão óssea isolada com aquela realizada juntamente com a remoção de aproximadamente 1 centímetro cúbico de gordura da região temporal inferior da órbita com o intuito de aumentar o retrocesso do bulbo ocular em nossas cirurgias. O resultado desta comparação demonstrou, no entanto, não haver aumento no retro-deslocamento do bulbo com esta nova abordagem. Porém, deve ser salientado que muitos fatores podem ter influído no resultado deste estudo retrospectivo. A quantidade de gordura retirada ter sido ainda insuficiente para a melhora dos resultados e fatores como os diferentes graus de fibrose e complacência de tecidos moles nas duas amostras de pacientes, bem como o tamanho de janela óssea realizadas ou até tratamentos clínico radiológicos associados em cada um dos grupos, pode ter interferido nos resultados. Acreditamos que novos estudos prospectivos de preferência envolvendo apenas pacientes com a forma lipogênica da orbitopatia distireoidiana serão importantes na definição do papel real da remoção de gordura associada à descompressão óssea da órbita.

\section{ABSTRACT}

Purpose: To compare the results of isolated antral-ethmoidal orbital decompression and that associated with orbital fat removal in patients with dysthyroid orbitopathy. Methods: Nineteen isolated antral-ethmoidal orbital decompressions were performed in a group of 12 patients (19 orbits) with the diagnosis of dysthyroid ophthalmopathy in the quiescent stage. The same procedure, but in association with orbital fat removal, were performed in another group of 8 patients (10 orbits) also with the diagnosis of dysthyroid ophthalmopathy in the quiescent stage. The results of both groups were compared. Results: In the first group the amount of retroplacement of the globe achieved $1-5 \mathrm{~mm}$ (mean $3.68 \pm 1.10 \mathrm{~mm}$ ) and in the second group it achieved 1-5 mm (mean 3.25 $\pm 1.36 \mathrm{~mm}$ ). Conclusions: There was no significant difference between the results of the isolated antral-ethmoidal orbital decompression and that associated with orbital fat removal. The removal of $1 \mathrm{ml}$ of seems not to 
improve the retroplacement of the globe as an association with a 2-wall orbital decompression. However, many conditions must be considered to conclude any comparison between the two orbital decompression techniques, such as orbital soft tissue compliance, amount of fat removal and size of bone opening.

Keywords: Graves' disease; Decompression, surgical/methods; Orbit/surgery; Ethmoid bone/surgery; Lipectomy; Comparative study; Retrospective studies

\section{REFERÊNCIAS}

1. Myron T. Orbital decompression for Graves' orbitopathy. In: Bosniak S. Principles and practice of ophthalmic plastic and reconstructive surgery. Philadelphia: W. B. Saunders; 1996.

2. Monteiro MLR. Oftalmopatia de Graves. In: Dantas AM, Monteiro MLR. Doenças da órbita. Rio de Janeiro: Cultura Médica; 2002.

3. Monteiro MLR, Cruz AAV, Manso PG. Orbitopatia distiroidiana. In: Soares EJC, Moura EM, Gonçalves JOR. Cirurgia plástica ocular. São Paulo: Roca; 1997.

4. Goldberg RA, Weinberg DA, Shorr N, Wirta D. Maximal, three-wall, orbital decompression through a coronal approach. Ophthalmic Surg Lasers. 1997; 28(10):832-43.

5. Olivari N. Transpalpebral decompression of endocrine ophthalmopathy (Graves'disease) by removal of intraorbital fat: an experience with 147 operations over 5 years. Plast Reconstr Surg. 1991;87(4):627-43; discussion 642-3.

6. Monteiro MLR, Ostroscki MR, Silva ALB, Bloise W. Descompressão orbitária antro-etmoidal na orbitopatia distiroidiana. Arq Bras Oftalmol. 2001;64(3): 189-94.

7. McCord CD Jr. Orbital decompression for Graves' disease. Exposure through lateral canthal and inferior fornix incision. Ophthalmology. 1981;88(6):533-41.
8. Siracuse-Lee DE, Kazim M. Orbital decompression: current concepts. Curr Opin Ophthalmol. 2002;13(5):310-6.

9. Trokel SL, Jakobiec FA. Correlation of CT scanning and pathologic features of ophthalmic Graves' disease. Ophthalmology. 1981;88(6):553-64.

10. Kazim M, Trokel SL, Acaroglu G, Elliot A. Reversal of dysthyroid optic neuropathy following orbital fat decompression. Br J Ophthalmol. 2000;84(6):600-5.

11. Cruz AAV, Leme VR. Orbital decompression: a comparison between transfornix/transcaruncular inferomedial and coronal inferomedial plus lateral approaches. Ophthal Plast Reconstr Surg. 2003;19(6):440-5; discussion 445.

12. Kazim M. Commentary on "Orbital Decompression". Ophthalmol Plast Reconstr Surg. 2003;19(6):445.

13. Van der Waal KG, de Visscher JG, Boukes RJ, Smeding B. Surgical treatment of Graves orbitopathy: a modified balanced technique. Int J Oral Maxillofac Surg. 2001;30(4):254-8.

14. McCord CD Jr. Current trends in orbital decompression. Ophthalmology. 1985;92(1):21-33.

15. Trokel S, Kazim M, Moore S. Orbital fat removal. Decompression for Graves orbitopathy. Ophthalmology. 1993;100(5):674-82.

16. Adenis JP, Robert PY, Lasudry JG, Dalloul Z. Treatment of proptosis with fat removal orbital decompression in Graves ophthalmopathy. Eur J Ophthalmol. 1998;8(4):246-52.

17. Shore JW, Carvajal J, Westfall CT. Miniplate reconstruction of the lateral orbital rim after orbital decompression for Graves disease. Ophthalmology. 1992;99(9):1433-9.

18. Goldberg RA, Kim AJ. The lacrimal keyhole, orbital doorjamb and basin of the inferior orbital fissure. The three areas of deep bone in the lateral orbit. Arch Opthalmol. 1998;116(2):1618-24.

19. Paridaens DA, Verhoeff K, Bouwens D, Van Den Bosch WA. Transconjuntival orbital decompression in Graves' ophthalmopathy: lateral approach ab interno. Br J Ophthalmol. 2000;84(7):775-81.

20. Mourits MP, Koornneef L, Wiersinga WM, Prummel MF, Berghout A, Van der Gaag R. Orbital decompression for Graves's ophthalmopathy by inferomedial, by infero-medial plus lateral, and by coronal approach. Ophthalmology. 1990;97(5):636-41.

21. Goldberg RA, Perry JD, Hortaleza V, Tong JT. Strabismus after balanced medial plus lateral wall only orbital decompression for dysthyroid orbitopathy. Ophthalmic Plast Reconstr Surg. 2000;16(4):271-7.

\section{Simpósio Cearense de Oftalmologia}

\section{7 a 19 de Novembro}

Fortaleza - CE

\section{IN FO RMAÇÕES: ARX Produções e Eventos}

Tel. (85) 4011-1572

E-mail: oftalmo@arxweb.com.br 